Monatsschr Kinderheilkd 2014 · 162:861-861

DOI 10.1007/s00112-014-3094-9

Online publiziert: 3. Oktober 2014

(c) Springer-Verlag Berlin Heidelberg 2014

\title{
G. Hansen
}

Klinik für Pädiatrische Pneumologie, Allergologie und Neonatologie,

Zentrum Kinderheilkunde und Jugendmedizin, Medizinische Hochschule Hannover

\section{Nahrungsmittelallergien}

Nahrungsmittelallergien gehören mit einer Prävalenz von 4-8\% zu den häufigsten Erkrankungen im Kindesalter. Schon der Verzehr einer sehr geringen Menge eines sehr potenten Allergens, wie z. B. Erdnuss, kann zu lebensbedrohlichen allergischen Reaktionen führen. Nahrungsmittel sind im Kindesalter die häufigsten Auslöser für Anaphylaxien, und für manche Patienten ist das regelmäßige Mitführen eines Notfallsets dauerhaft erforderlich. Während manche Nahrungsmittelallergien wie Kuhmilch- und Hühnereiallergien oft sehr früh auftreten und sich häufig bis ins Schulkindalter wieder verlieren, tolerieren Kinder mit Erdnussoder Nussallergie diese Lebensmittel auch später nicht gut.

Die therapeutischen Möglichkeiten bei Nahrungsmittelallergie sind begrenzt. Die Eliminationsdiät nach ausführlicher Beratung bleibt das entscheidende Fundament. In den letzten Jahren wurden die orale Immuntherapie (OIT) und die sublinguale Immuntherapie (SLIT) als kausale Therapieoption für Nahrungsmittelallergien untersucht und haben neue Hoffnungen geweckt. Die Zukunft wird zeigen, ob diese Hoffnungen erfüllt werden können.
Häufig werden Nahrungsmittelallergien und die atopische Dermatitis (AD) in Zusammenhang gebracht. Sie können gleichzeitig auftreten, und der Verzehr bestimmter Nahrungsmittel kann mit der Auslösung bzw. der Verschlechterung von Ekzemen einhergehen. In dem vorliegenden Leitthemenheft der Monatsschrift Kinderheilkunde wird von sehr erfahrenen Spezialisten aus Berlin, Hamburg und Hannover über dieses wichtige Thema berichtet. Ich wünsche Ihnen viel Freude beim Lesen!

Ihre

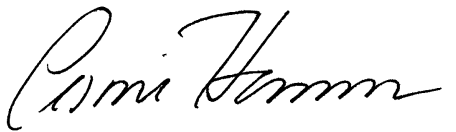

Gesine Hansen

\section{Korrespondenzadresse}

\section{Prof. Dr. G. Hansen}

Klinik für Pädiatrische Pneumologie, Allergologie und Neonatologie, Zentrum

Kinderheilkunde und Jugendmedizin, Medizinische Hochschule Hannover Carl-Neuberg-Str. 1, 30625 Hannover hansen.gesine@mh-hannover.de

\section{Einhaltung ethischer Richtlinien}

Interessenkonflikt. G. Hansen gibt an, dass kein Interessenkonflikt besteht. 